\title{
New records of Dermestidae (Coleoptera) for Bali Island, Indonesia
}

\section{Новые находки зкуков-кожкеедов (Coleoptera: Dermestidae) с острова Бали, Индонезия}

\author{
J. Háva*, N. Suprayitno** \\ И. Гава*, Н. Супраетно**
}

\author{
* Forestry and Game Management Research Institute, Strnady 136, Praha 5 - Zbraslav CZ-156 00 Czech Republic. E-mail: \\ jh.dermestidae@volny.cz. \\ * Научно-исследовательский институт охотничьего хозяйства, Стрнады 136, Прага 5 - Збраслав CZ-156 00 Чешская \\ Республика. \\ ** Nano Tours, Ubud, Bali, Indonesia. \\ ** Нано Турс, Убуд, Бали, Индонезия.
}

Key words: Faunistics, new record, Thorictodes, Thaumaglossa, Indonesia, Bali Island.

Ключевые слова: Фаунистика, новые находки, Thorictodes, Thaumaglossa, Индонезия, Бали.

Abstract. Three dermestid beetle species, Thorictodes dartevellei John, 1961, Thaumaglossa rufula Pic, 1931 and Thaumaglossa petrstanda Háva, 2003, are newly recorded from Indonesia, Bali Island.

Резюме. Впервые на острове Бали зарегистрированы Thorictodes dartevellei John, 1961, Thaumaglossa rufula Pic, 1931 и Thaumaglossa petrstanda Háva, 2003.

\section{Introduction}

The beetle family Dermestidae currently contains about 1690 species and subspecies worldwide [Háva, $2015,2020]$. The family is recently being studied more extensively, and more articles are being published about the species from the Oriental Region. Dermestidae from Bali Island are poorly known, this work contains the first records for three species for this island, one of them is a new record for Indonesia at the same time.

\section{Material and Methods}

Nomenclature and systematics in this paper follow Háva [2015]. All species were discovered on Bali by the senior author, and were determined by Jiří Háva based on photographs made and digitally communicated by Suprayitno. The work in Bali uses a workflow based on a locally made mobile phone with a macro lens attached to it; and then communication with international taxonomic expert via social media tools such as WhatsApp and Facebook. This approach was in detail described by Suprayitno et al. [2017].

The material is deposited in the private collection of Nano Suprayitno, Sukuwati, Bali, Indonesia. The beetles will later be moved to Museum Zoologicum
Bogoriense (Cibinong, West Java) for permanent storage.

\section{Results}

\author{
Thorictinae \\ Thaumaphrastini \\ Thorictodes dartevellei John, 1961
}

Fig. 1.

Material. Indonesia, Bali Island, Gianyar, Sukawati, Batubulan, Subak Blaki street, 6.04.2020, N. Supravitno leg. et coll., 1 spec., J. Háva det. The specimen was collected afternoon after heavy rain, found on house floor.

Distribution. Species known from England (introduced), DR Congo, China: Yunnan (introduced), India (introduced) [Háva, 2015], Philippines: Mindanao (introduced) [Háva, Herrmann, 2017], New to Indonesia: Bali Island.

\section{Megatominae Megatomini Thaumaglossa rufula Pic, 1931} Fig. 2.

Material. Indonesia, Bali Island, Gianyar, Sukawati, Batubulan, Subak Blaki street, 16.04.2020, N. Suprayitno leg.

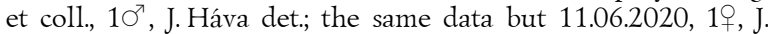
Háva det.

Distribution. Species known from the Philippines and Indonesia: Java, Kalimantan [Háva, 2015], new to Bali Island.

\section{Thaumaglossa petrstanda Háva, 2003 \\ Figs. 3-4.}

Material. Indonesia, Bali Island, Kabupaten Gianyar, Kec. Sukawati, Batubulan Kangin, SMP Negeri 3 Sukawati, 19.04.2020, N. Suprayitno leg. et coll., collected from a Mantis ootheca (Fig. 4), 1이, J. Háva det. 

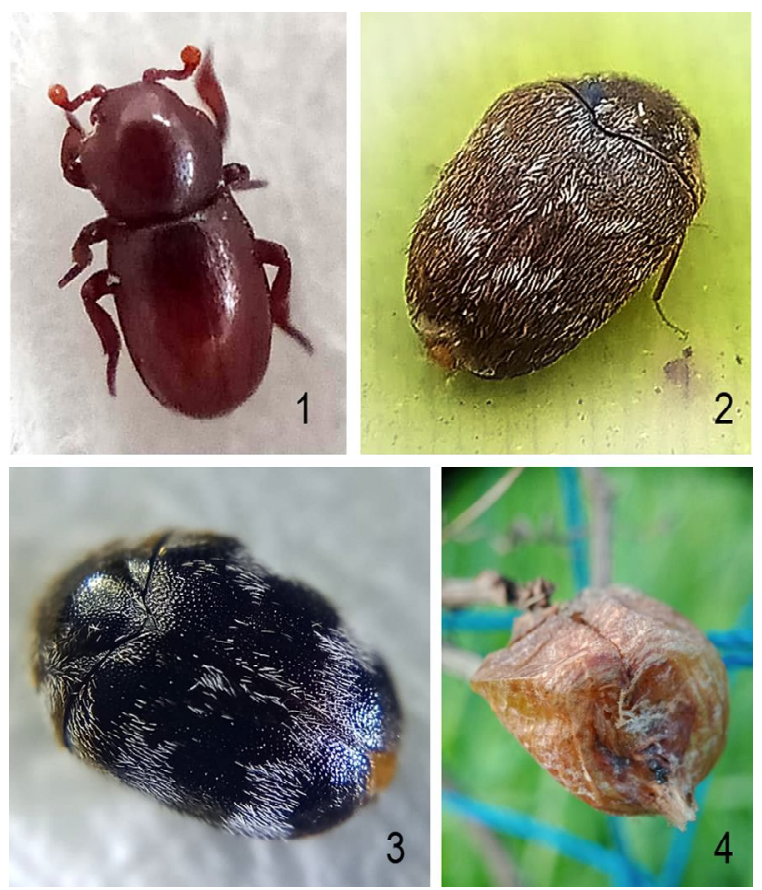

Figs 1-4. Dermestidae. 1 - Thorictodes dartevellei, habitus, 2 - Thaumaglossa rufula, habitus, 3 - Thaumaglossa petrstanda, habitus, 4 - mantis ootheca, where Thaumaglossa petrstanda was collected.

Рис. 1-4. Dermestidae. 1 - Thorictodes dartevellei, внешний виА, 2 - Thaumaglossa rufula, внешний виА, 3 Thaumaglossa petrstanda, внешний виА, 4 - оотека богомола, гАе бым собран Thaumaglossa petrstanda.

Distribution. Species known from Indonesia: Sumba [Háva, 2015], new to Bali Island.

Remarks. The mentioned species was collected in Sukawati, Batubulan together with Attagenus (Attagenus) fasciatus (Thunberg, 1795), Orphinus (Orphinus) fulvipes (GuérinMéneville, 1838), Thaumaglossa rufocapillata Redtenbacher, 1867 and Evorinea iota (Arrow, 1915), species Attagenus (Aethriostoma) undulatus (Motschulsky, 1858) was collected at Lake Batur, Kintamani.

\section{List of Dermestidae recorded from Bali Island}

\author{
Dermestinae
}

Dermestes (Dermestinus) maculatus DeGeer, 1774

Thorictinae

Thorictodes dartevellei John, 1961

Trinodinae

Evorinea iota (Arrow, 1915)

\section{Attageninae}

Attagenus (Attagenus) fasciatus (Thunberg, 1795)

Attagenus (Aethriostoma) undulatus (Motschulsky, 1858)

\section{Megatominae}

Orphinus (Orphinus) baliensis Háva, 2016

Orphinus (Orphinus) fulvipes (Guérin-Méneville, 1838)

Thaumaglossa petrstanda Háva, 2003

Thaumaglossa rufocapillata Redtenbacher, 1867

Thaumaglossa rufula Pic, 1931

\section{Acknowledgements}

The paper was supported by the Ministry of Agriculture of the Czech Republic, institutional support MZE-RO0118.

\section{References}

Háva J. 2015. World Catalogue of Insects. Vol.13. Dermestidae (Coleoptera). Leiden-Boston: Brill, xxvi +419 p.

Háva J. 2020. Dermestidae World (Coleoptera). World Wide Web electronic publication (open in 2004): http:// www.dermestidae.wz.cz (version 2018, update January 2020)

Háva J., Herrmann A. 2017. New faunistic records and remarks on Dermestidae (Coleoptera) - Part $16 / /$ Folia Heyrovskyana. Ser.A. Vol.25. No.2. P.4-4.

Suprayitno N., Narakusumo R.P., Von Rintelen T., Hendrich L., Balke M. 2017. Taxonomy and Biogeography without frontiers // WhatsApp, Facebook and smartphone digital photography let citizen scientists in more remote localities step out of the dark. Biodivers Data J: e19938. doi:10.3897/ BDJ.5.e19938 